\title{
SUMS OF LATTICE HOMOMORPHISMS
}

\author{
S. J. BERNAU, C. B. HUIJSMANS, AND B. DE PAGTER
}

(Communicated by Louis J. Ratliff, Jr.)

\begin{abstract}
Let $E$ and $F$ be Riesz spaces and $T_{1}, T_{2}, \ldots, T_{n}$ be linear lattice homomorphisms (henceforth called lattice homomorphisms) from $E$ to $F$. If $T=\sum_{i=1}^{n} T_{i}$, then it is easy to check that $T$ is positive and that if $x_{0}, x_{1}, \ldots x_{n} \in E$ and $x_{i} \wedge x_{j}=0$ for all $i \neq j$, then $\bigwedge_{i=0}^{n} T x_{i}=0$. The purpose of this note is to show that if $F$ is Dedekind complete, the above necessary condition for $T$ to be be the sum of $n$ lattice homomorphisms is also sufficient. The result extends to sums of disjointness preserving operators, thereby leading to a characterization of the ideal of order bounded operators generated by the lattice homomorphisms.
\end{abstract}

Throughout this note we assume that $E$ and $F$ are real Riesz spaces. For unexplained terminology we refer the reader to references $[1,6,8,9]$.

Definition 1. Let $n$ be a positive integer and $T$ be a linear operator from $E$ to $F$. We say that $T$ is $n$-disjoint if $T$ is order bounded, and for all $x_{0}, x_{1}, \ldots, x_{n} \in E$ such that $\left|x_{i}\right| \wedge\left|x_{j}\right|=0$ for all $i \neq j$, we have $\bigwedge_{i=0}^{n}\left|T x_{i}\right|$ $=0$.

Clearly a 1-disjoint positive linear operator from $E$ to $F$ is a lattice homomorphism, and a general 1-disjoint linear operator is order bounded and disjointness preserving (i.e., if $|u| \wedge|v|=0$, then $|T u| \wedge|T v|=0$ ). We can readily check that every (positive) linear operator on $\mathbb{R}^{n}$ is the sum of $n$ (lattice homomorphisms) disjointness preserving operators. To see this take the matrix representation relative to the standard basis of $\mathbb{R}^{n}$ (or any positive pairwise disjoint basis if greater generally is wanted), and write the matrix as the sum of $n$ matrices each with at most one nonzero column.

Before stating our first result we recall some facts about disjointness preserving operators. These can be found in [2,4]. Note, however, that the term disjointness preserving in [2] has a different definition and that [2] considers self-maps of $E$ rather than maps from $E$ to $F$. The relevant proofs in [2] all take place in the range of the operator and apply without change. The results we need are given by the following:

Received by the editors October 16, 1990.

1980 Mathematics Subject Classification (1985 Revision). Primary 46A40, 06F20.

Key words and phrases. Lattice homomorphism, disjointness preserving operator, $n$-disjoint operator, Dedekind complete Riesz space, band projection, minimal positive extension.

Presented to the Society, January 18, 1991 in San Francisco. 
Theorem $[2,4,7]$. Let $T$ be an order bounded linear operator from a Riesz space $E$ into an archimedean Riesz space $F$ such that $|T u| \wedge|T v|=0$ for all $u, v \in E$ with $|u| \wedge|v|=0$. Then there exist lattice homomorphisms $T^{+}, T^{-}$, and $|T|$ from $E$ to $F$ such that $T=T^{+}-T^{-},\left(T^{+}\right) x=(T x)^{+}$and $\left(T^{-}\right) x=(T x)^{-}$ $(0 \leq x \in E),|T|=T^{+}+T^{-}$, and $|T x|=|T|(|x|)(x \in E)$.

Proposition 2. Let $F$ be archimedean and $T_{1}, T_{2}, \ldots, T_{n}$ be order bounded and disjointness preserving from $E$ to $F$. If $T=\sum_{i=1}^{n} T_{i}$, then $T$ is $n$ disjoint. The archimedean assumption on $F$ can be dropped if $T_{1}, T_{2}, \ldots, T_{n}$ are assumed to be positive (and hence lattice homomorphisms).

Proof. Suppose $x_{0}, x_{1}, \ldots, x_{n} \in E$ and $\left|x_{i}\right| \wedge\left|x_{j}\right|=0$ for all $i \neq j$. Then

$$
\bigwedge_{i=0}^{n}\left|T x_{i}\right| \leq \sum\left|T_{i_{0}} x_{0}\right| \wedge\left|T_{i_{1}} x_{1}\right| \wedge \cdots \wedge\left|T_{i_{n}} x_{n}\right|
$$

where the summation is over all choices of $i_{0}, i_{1}, \ldots, i_{n}$ from $\{1,2, \ldots, n\}$. In each summand at least two of the subscripts $i_{0}, i_{1}, \ldots, i_{n}$ are identical. Suppose we have $k \neq l$ and $i_{k}=i_{1}=j$. Then

$$
\begin{aligned}
\left|T_{i_{0}} x_{0}\right| \wedge\left|T_{i_{1}} x_{1}\right| \wedge \cdots \wedge\left|T_{i_{n}} x_{n}\right| \leq\left|T_{j} x_{k}\right| \wedge\left|T_{j} x_{l}\right| & =\left(\left|T_{j}\right|\left(\left|x_{k}\right|\right)\right) \wedge\left(\left|T_{j}\right|\left(\left|x_{l}\right|\right)\right) \\
& =\left|T_{j}\right|\left(|x|_{k} \wedge\left|x_{l}\right|\right)=0 .
\end{aligned}
$$

It follows that $\bigwedge_{i=0}^{n}\left|T x_{i}\right|=0$ as required The archimedean property of $F$ is used only for the existence and properties of the $\left|T_{j}\right|$, so it need not be assumed when the $T_{j}$ are all lattice homomorphisms.

We now consider sufficiency. In this connection we note that Huijsmans and de Pagter [5, Remark 2.3] ask if a 2-disjoint positive operator is the sum of two lattice homomorphisms and state that this property characterizes such operators if $F=\mathbf{C}(X)$, with $X$ extremally disconnected. We begin with the standard description of the minimal positive extension of the restriction of $T$ to the solid subspace of $E$ generated by $u$ [9, Theorem 83.8].

Lemma 3. Let $E$ and $F$ be Riesz spaces with $F$ Dedekind complete. Suppose that $T$ is a positive linear map from $E$ into $F$ and $0 \leq u \in E$. If $T_{u}: E \rightarrow F$ is defined by

$$
T_{u} x=\bigwedge_{n=1}^{\infty} T(x \wedge n u) \quad(0 \leq x \in E)
$$

and $T_{u} x=T_{u}\left(x^{+}\right)-T_{u}\left(x^{-}\right)$for arbitrary $x \in E$, then $T_{u}$ is linear from $E$ to $F$, dominated by $T$ on $E$, annihilates the disjoint complement of $u$, and agrees with $T$ on the ideal generated by $u$. In the case that $E=F$ and $T$ is the identity, then $T_{u}$ is the band projection on $u^{d d}$.

Lemma 4. Let $E$ and $F$ be Riesz spaces with $F$ Dedekind complete and $T$ a positive n-disjoint linear operator from $E$ to $F$. Suppose $u_{0}, u_{1}, \ldots, u_{n-1} \in E$ and $u_{i} \wedge u_{j}=0$ for all $i \neq j$. Let $P_{i}$ denote the band projection of $F$ onto $T u_{i}^{d d}$, and write $T_{0}$ for the operator $T_{u_{0}}$, defined as in (1) above. Define $R=P_{0} P_{1} \ldots P_{n-1} T_{0}$ and $S=P_{0} P_{1} \ldots P_{n-1}\left(T-T_{0}\right)$. Then $R$ is a lattice homomorphism, and $S$ is positive and $(n-1)$-disjoint.

Proof. It is clear that $R$ and $S$ are linear and positive. Suppose $x, y \in E$ and $x \wedge y=0$. For all positive integers $m, u_{1}, u_{2}, \ldots, u_{n-1}, x \wedge m u_{0}$, 
$y \wedge m u_{0}$ are $n+1$ mutually pairwise disjoint positive elements of $E$. Hence $T u_{1} \wedge T u_{2} \wedge \cdots \wedge T u_{n-1} \wedge T\left(x \wedge m u_{0}\right) \wedge T\left(y \wedge m u_{0}\right)=0$. Taking supremum over $m$ we conclude that $T u_{1} \wedge T u_{2} \wedge \cdots \wedge T u_{n-1} \wedge T_{0} x \wedge T_{0} y=0$, from which it follows that

$$
0=P_{1} P_{2} \ldots P_{n-1}\left(T_{0} x \wedge T_{0} y\right)=\left(P_{1} P_{2} \ldots P_{n-1} T_{0} x\right) \wedge\left(P_{1} P_{2} \ldots P_{n-1} T_{0} y\right) .
$$

Since $P_{0}$ is also a lattice homomorphism, we have $R x \wedge R y=0$, so $R$ is a lattice homomorphism as claimed

Now suppose that $x_{1}, x_{2}, \ldots, x_{n}$ are $n$ mutually disjoint positive elements of $E$. It is sufficient to show that

$$
\bigwedge_{i=1}^{n} P_{0}\left(T-T_{0}\right) x_{i}=0 .
$$

Let $l$ be a positive integer. Write $x=x_{1}+x_{2}+\cdots+x_{n}$, and observe that $\left(u_{0}-u_{0} \wedge(1 / l) x\right)$, and the $n$ elements $x_{i}-x_{i} \wedge l u_{0}$, are $n+1$ mutually disjoint positive elements of $E$ (for each $i, u_{0}-u_{0} \wedge(1 / l) x \leq u_{0}-u_{0} \wedge(1 / l) x_{i}=$ $\left.(1 / l)\left(l u_{0}-x_{i} \wedge l u_{0}\right)\right)$. It follows that

$$
\begin{aligned}
T u_{0} \wedge \bigwedge_{i=1}^{n}\left(T-T_{0}\right) x_{i} \leq & T u_{0} \wedge \bigwedge_{i=1}^{n} T\left(x_{i}-x_{i} \wedge l u_{0}\right) \\
\leq & \left(T\left(u_{0}-(1 / l) u_{0} \wedge x\right)\right) \wedge \bigwedge_{i=1}^{n} T\left(x_{i}-x_{i} \wedge l u_{0}\right) \\
& +(1 / l) T\left(u_{0} \wedge x\right) \wedge \bigwedge_{i=1}^{n} T\left(x_{i}-x_{i} \wedge l u_{0}\right) \\
= & 0+(1 / l) T\left(u_{0} \wedge x\right) \wedge \bigwedge_{i=1}^{n} T\left(x_{i}-x_{i} \wedge l u_{0}\right) \leq(1 / l) T u_{0}
\end{aligned}
$$

Since this is true for all $l$, we have

$$
T u_{0} \wedge \bigwedge_{i=1}^{n}\left(T-T_{0}\right) x_{i}=0
$$

and hence

$$
0=P_{0} \bigwedge_{i=1}^{n}\left(T-T_{0}\right) x_{i}=\bigwedge_{i=1}^{n} P_{0}\left(T-T_{0}\right) x_{i}
$$

Theorem 5. Let $E$ and $F$ be Riesz spaces with $F$ Dedekind complete and $T$ a positive $n$-disjoint linear operator from $E$ to $F$. Then there exist $n$ lattice homomorphisms, $T_{1}, T_{2}, \ldots T_{n}$, from $E$ to $F$ such that $T=\sum_{i=1}^{n} T_{i}$.

Proof. The proof is by induction on $n$. The case $n=1$ is a tautology.

Suppose the theorem is true for $n-1$. By Zorn's lemma there is a maximal set $\mathscr{P}$ of band projections on $F$ such that if $P_{1}, P_{2} \in \mathscr{P}$ and $P_{1} \neq$ $P_{2}$, then $P_{1} P_{2}=0$; and for each $P \in \mathscr{P}$ there exist $n$ mutually disjoint positive elements $u_{0}, u_{1}, \ldots, u_{n-1} \in E$ such that the range, $P F$, of $P$ is contained in $\left(T u_{0} \wedge T u_{1} \wedge \cdots \wedge T u_{n-1}\right)^{d d}$. By Lemma 4, for each $P \in \mathscr{P}$, there exists a 
lattice homomorphism $T_{1}(P)$ such that $T_{1}(P)=P T_{1}(P)$ and $P T-T_{1}(P)$ is $(n-1)$ - disjoint. For $0<x \in E$, define

$$
T_{1} x=\bigvee\left\{T_{1}(P) x: P \in \mathscr{P}\right\} .
$$

If, $x, y \in E$ and $x \wedge y \leq 0$, then we have

$$
\begin{aligned}
T_{1}(x+y) & =\bigvee\left\{T_{1}(P)(x+y): P \in \mathscr{P}\right\} \\
& \leq \bigvee\left\{T_{1}(P) x+T_{1}(Q) y: P, Q \in \mathscr{P}\right\} \\
& =\bigvee\left\{T_{1}(P) x: P \in \mathscr{P}\right\}+\bigvee\left\{T_{1}(Q) y: Q \in \mathscr{P}\right\} \\
& =T_{1} x+T_{1} y .
\end{aligned}
$$

If $P, Q \in \mathscr{P}$ and $P \neq Q$, then $T_{1}(P) x \wedge T_{1}(Q) y=0$, and hence

$$
T_{1}(P) x+T_{1}(Q) y=T_{1}(P) x \vee T_{1}(Q) y \leq T_{1}(P)(x+y) \vee T_{1}(Q)(x+y) .
$$

Thus

$$
T_{1} x+T_{1} y \leq \bigvee\left\{T_{1}(P)(x+y): P \in \mathscr{P}\right\}=T_{1}(x+y),
$$

and we have equality.

It follows that $T_{1}$ extends naturally to a linear operator from $E$ to $F$. Suppose that $x, y \in E$ and $x \wedge y=0$. Then

$$
\begin{aligned}
T_{1} x \wedge T_{1} y & =\bigvee\left\{T_{1}(P) x: P \in \mathscr{P}\right\} \wedge \bigvee\left\{T_{1}(Q) y: Q \in \mathscr{P}\right\} \\
& =\bigvee\left\{T_{1}(P) x \wedge T_{1}(Q) y: P, Q \in \mathscr{P}\right\} \\
& =\bigvee\left\{T_{1}(P) x \wedge T_{1}(P) y: P \in \mathscr{P}\right\} \\
& =\bigvee\left\{T_{1}(P)(x \wedge y): P \in \mathscr{P}\right\}=0
\end{aligned}
$$

Thus $T_{1}$ is a lattice homomorphism.

Let $S=T-T_{1}$. We will complete the proof by showing that $S$ is $(n-1)$ disjoint. To this end suppose that $u_{0}, u_{1}, \ldots, u_{n-1} \in E$ and $u_{i} \wedge u_{j}=0$ for all $i \neq j$. By maximality of $\mathscr{P}$ we see that the restriction of $\bigvee\{P: P \in \mathscr{P}\}$ to $\left(T u_{0} \wedge T u_{1} \wedge \cdots \wedge T u_{n-1}\right)^{d d}$ is the identity. Since $S u_{0} \wedge S u_{1} \wedge \cdots \wedge S u_{n-1} \in$ $\left(T u_{0} \wedge T u_{1} \wedge \cdots \wedge T u_{n-1}\right)^{d d}$,

$$
\begin{aligned}
S u_{0} \wedge S u_{1} \wedge \cdots \wedge S u_{n-1} & =\bigvee\left\{P\left(S u_{0} \wedge S u_{1} \wedge \cdots \wedge s u_{n-1}\right): P \in \mathscr{P}\right\} \\
& =\bigvee\left\{P S u_{0} \wedge P S u_{1} \wedge \cdots \wedge P S u_{n-1}: P \in \mathscr{P}\right\} \\
& =0
\end{aligned}
$$

because each $P S=P T-T_{1}(P)$ is $(n-1)$-disjoint.

The proof is complete.

Theorem 6. Let $E$ and $F$ be Riesz spaces with $F$ Dedekind complete and $T$ an n-disjoint linear operator from $E$ to $F$. Then there exist $n$ disjointness preserving linear operators, $T_{1}, T_{2}, \ldots, T_{n}$, from $E$ to $F$ such that $T=\sum_{i=1}^{n} T_{i}$. Proof. First we prove that $|T|$ is $n$-disjoint (of course it is positive). For this, note that $|T|(|x|)=\bigvee\{|T u|: u \in E$ and $|u| \leq|x|\}$ for all $x \in E$. Suppose 
$x_{0}, x_{1}, \ldots, x_{n} \in E$ and $x_{i} \wedge x_{j}=0$ for all $i \neq j$. If $u_{j} \in E$ and $\left|u_{j}\right| \leq x_{j}$ for each $j$, then the $u_{j}$ are $n+1$ mutually disjoint elements of $E$, so

$$
\bigwedge_{j=0}^{n}\left|T u_{j}\right|=0
$$

Taking suprema for successive values of $j$ over all $u_{j} \in E$ such that $\left|u_{j}\right| \leq\left|x_{j}\right|$, we obtain $\bigwedge_{j=0}^{n}|T| x_{j}=0$, as required.

By Theorem 5, there exist lattice homomorphisms $S_{1}, S_{2}, \ldots, S_{n}$ such that $|T|=S_{1}+S_{2}+\cdots+S_{n}$. Since $0 \leq T^{+} \leq|T|$ and $0 \leq T^{-} \leq|T|$, the Riesz decomposition property shows that there exists order bounded linear operators $U_{1}, U_{2}, \ldots, U_{n}, V_{1}, V_{2}, \ldots, V_{n}$ from $E$ to $F$ such that $T^{+}=U_{1}+U_{2}+$ $\cdots+U_{n}$ and $T^{-}=V_{1}+V_{2}+\cdots+V_{n}$. For each $i$ write $T_{i}=U_{i}-V_{i}$. Since $\left|T_{i}\right| \leq U_{i}+V_{i} \leq 2 S_{i}$, it is clear that $T_{i}$ is disjointness preserving for each $i$. By construction $T=T_{1}+T_{2}+\cdots+T_{n}$, so the proof is complete.

The method of proof makes it clear that there is no hope of proving a uniqueness theorem for the decomposition of an $n$-disjoint linear operator as the sum of $n$ lattice homomorphisms, and it also shows how to construct counterexamples.

Example 7. Let $E=F=\mathbb{R}^{2}$, and define $T$ by $T(a, b)=(a+b, a+b)$. Then $T$ is 2-disjoint (by default since $\operatorname{dim} E=2$ ). Now let $I$ be the identity map of $E$ to $F$, and define $R$ from $E$ to $F$ by $R(a, b)=(a, a)$. It is easy to check that $I$ and $T-I$ are lattice homomorphisms, both of rank 2 , and that $R$ and $T-R$ are lattice homomorphisms, both of rank 1. In fact, this example can be easily modified to apply to any $T \in L\left(\mathbb{R}^{2}\right)$ of the form $T(a, b)=(\alpha a+\beta b$, $\gamma a+\delta b)$ with $\alpha, \beta, \gamma$, and $\delta$ all positive. The relevant equalities are

$$
a(\alpha, \gamma)+b(\beta, \delta)=(\alpha a+\beta b, \gamma a+\delta b)=(\alpha a, \delta b)+(\beta b, \gamma a) .
$$

So far our perspective has been local. We close with a few global remarks. Let us consider the ideal $\mathscr{L}$ of order bounded operators generated by the lattice homomorphisms. Clearly $\mathscr{L}$ is the set of order bounded linear $T$ such that there exist a positive integer $n$ and $n$ lattice homomorphisms $S_{1}, S_{2}, \ldots, S_{n}$ such that $|T| \leq \sum_{i=1}^{n} S_{i}$. Thus we may restate Theorem 6 as follows:

Theorem 8. Let $E$ and $F$ be Riesz spaces with $F$ Dedekind complete and $\mathscr{L}$ the ideal of order bounded linear operators from $E$ to $F$ generated by the lattice homomorphisms. Then $\mathscr{L}$ consists precisely of those order bounded linear operators that are n-disjoint for some positive integer $n$.

A type of order closure of $\mathscr{L}$ has been recently considered by Carothers and Feldman [3]. They call an order bounded linear operator $T$ a local homomorphism if there exists a net $\left\{M_{\alpha}\right\}$ of orthomorphisms increasing to the identity such that each $M_{\alpha} T$ is a finite sum of lattice homomorphisms. For $E=C(K)$ with $K$ compact Hausdorff, they characterize the local homomorphisms and extend this to Banach lattices when $E$ has a quasi-interior point. The closest overlap between their paper and ours comes in the following result, which they state with a promise of a proof to follow in a subsequent paper. 
Theorem 9. Let $X$ and $Y$ be compact Hausdorff spaces and $C(Y)$ be Dedekind complete. If $T$ is an order bounded linear operator from $X$ to $Y$ for which there exists a positive integer $n$ such that for each $y \in Y$ there exist nonnegative reals $a_{1}, a_{2}, \ldots, a_{n}$ and elements $x_{1}, x_{2}, \ldots, x_{n}$ in $X$ with the property that the evaluation functionals (denoted by a circumflex) satisfy

$$
T^{*} \hat{y}=\sum_{i=1}^{n} a_{i} \hat{x}_{i}
$$

then $T$ is a sum of $n$ lattice homomorphisms.

Proof. It is almost trivial to check if such an operator is $n$-disjoint. Theorem 5 then applies.

Example 1 in [3] also shows that some sort of order completeness of $F$ is necessary for our Theorem 5, and hence Theorems 6 and 8 , to hold. The example gives a 2-disjoint operator from $C([0,1])$ to $C([0,1])$ that is not a sum of two lattice homomorphisms. It would be interesting to have an example in which $F$ is $\sigma$-Dedekind complete and a 2-disjoint operator is still not the sum of two lattice homomorphisms. Our proof of Theorem 5 requires the full force of Dedekind completeness. We know of no weaker hypotheses under which the Theorem may be true.

Our thanks are due to C. D. Aliprantis for informing us of [3].

\section{REFERENCES}

1. C. D. Aliprantis and O. Burkinshaw, Positive operators, Academic Press, Orlando, 1985.

2. S. J. Bernau, Orthomorphisms of archimedean vector lattices, Math. Proc. Cambridge Philos. Soc. 89 (1981), 119-128.

3. David C. Carothers and William A. Feldman, Sums of homomorphisms on Banach lattices, J. Operator Theory (to appear).

4. Michel Duhoux and Mathieu Meyer, A new proof of the lattice structure of orthomorphisms, J. London Math. Soc. (2) 25 (1981), 375-378.

5. C. B. Huijsmans and B. de Pagter, Disjointness preserving and diffuse operators, Compositio Math. 79 (1991), 351-374.

6. W. A. J. Luxemburg and A. C. Zaanen, Riesz spaces. I, North-Holland, Amsterdam, 1971.

7. M. Meyer, Le stabilisateur d'un espace vectoriel réticulé, C. R. Acad Sci. Paris Sér. A 283 (1976), 249-250.

8. H. H. Schaefer. Banach lattices and positive operators. Grundlehren Math. Wiss., vol. 215, Springer, Berlin, 1974.

9. A. C. Zaanen, Riesz spaces. II, North-Holland, Amsterdam, 1983. 79968

Department of Mathematical Sciences, University of Texas at El Paso, El Paso, Texas

Department of Mathematics and Computer Science, University of Leiden, Niels Bohrweg 1, 2300 CA Leiden, The NetherLands

Department of Mathematics, Delft University of Technology, JulianalaAn 132, 2628 BL Delft, The Netherlands 